\title{
CARACTERÍSTICAS DAS GESTANTES ATENDIDAS EM CONSULTA DE ENFERMAGEM AMBULATORIAL DE PRÉ- NATAL: RETRATO DE UMA DÉCADA
}

\author{
CHARACTERISTICS OF PREGNANT WOMEN ATTENDED IN \\ PRENATAL OUTPATIENT NURSING CONSULTATION: PORTRAIT OF \\ $A D E C A D E$
}

\author{
Andressa Carlos Silva Gonçalves ${ }^{1}$ \\ Geane Silva Oliveira ${ }^{2}$ \\ Renata Lívia Silva Moreira Fonseca de Medeiros ${ }^{3}$ \\ Ocilma Barros de Quental ${ }^{4}$
}

INTRODUÇÃO: A gestação é um evento natural, constituído de inúmeras mudanças físicas, psicológicas e sociais. Atrelado à gestação, está um grande problema enfrentado pela saúde pública, a morbimortalidade materna, que embora esteja em crescente queda nos últimos anos, ainda é um agravo considerável. As unidades básicas de saúde são a porta de entrada do SUS, no tocante a prevenção, proteção e promoção da saúde. E dentro desse serviço, temos a assistência ao pré-natal, que busca através do acompanhamento contínuo assegurar um sadio decorrer do ciclo gestacional e proporcionar o nascimento de um bebê saudável. OBJETIVO: apresentar as características das gestantes atendidas em consultas de enfermagem ambulatoriais no período de 2011-2021. METODOLOGIA: O estudo trata-se de um levantamento retrospectivo, que foi realizado através de pesquisa documental. A mesma ocorreu por meio de consulta ao banco de dados do Sistema de Informação do Pré-natal (Sisprenatal), na Secretaria Municipal de Saúde de Umari-ce, no setor de Informática. Foi feita análise das seguintes variáveis número de gestantes cadastradas, faixa etária, raça/cor, classificação de risco, testes rápidos e exames. Posteriormente a coleta dos dados, ocorreu à elaboração das tabelas, contemplando as variáveis da pesquisa. Só após a ciência e concordância da Secretaria Municipal de Umari-Ce, o protocolo do estudo foi submetido à apreciação do Comitê de Ética em Pesquisa com Seres Humanos da Faculdade Santa, conforme Resolução CNS № 466 de 201 com parecer no 5.110 .876 de aprovação. RESULTADOS: A análise das variáveis mostrou deficiências na assistência prestada, principalmente com

\footnotetext{
${ }^{1}$ Graduanda de Enfermagem pela Faculdade Santa Maria- FSM, Cajazeiras, PB.

${ }^{2}$ Mestre, Docente da Faculdade Santa Maria- FSM, Cajazeiras, PB.

${ }^{3}$ Doutora, Docente da Faculdade Santa Maria- FSM, Cajazeiras, PB.

${ }^{4}$ Doutora, Docente da Faculdade Santa Maria- FSM, Cajazeiras, PB.
} 
relação à captação precoce e realização de exames. Considerando a amostra, $65,5 \%$ foram cadastradas até a $12^{\text {a }}$ semana de gestação, $47 \%$ foram submetidas a seis ou mais consultas, 34\% referiram idade acima de 34 anos, 62\% foram classificadas dentro dos parâmetros para alto risco gestacional. Os resultados apontaram ausência no quesito "exames solicitados no terceiro trimestre" para o grupo estudado. CONCLUSÃO: O estudo revela fragilidade na captação precoce das gestantes para início do acompanhamento pré-natal, retratando a presença de alto risco entre a amostra, assim como discrepância entre os componentes solicitação e realização de testes rápidos e exames laboratoriais.

PALAVRAS CHAVE: Gravidez; Cuidado pré-natal; Cuidados de enfermagem; Enfermagem Obstétrica; Atenção Primária à Saúde;

INTRODUCTION: Pregnancy is a natural event, consisting of numerous physical, psychological and social changes. Linked to pregnancy, there is a major problem faced by public health, maternal morbidity and mortality, which, although decreasing in recent years, is still a considerable problem. Basic health units are the gateway to the SUS in terms of prevention, protection and health promotion. And within this service, we have prenatal care, which seeks, through continuous monitoring, to ensure a healthy course of the gestational cycle and provide for the birth of a healthy baby. OBJECTIVE: to present the characteristics of pregnant women attended in outpatient nursing consultations in the period 2011-2021. METHODOLOGY: The study is a retrospective survey, which was carried out through documentary research. The same occurred through consultation of the database of the Prenatal Information System (Sisprenatal), at the Municipal Health Department of Umari-ce, in the Information Technology sector. The following variables were analyzed: number of registered pregnant women, age group, race/color, risk classification, rapid tests and exams. After data collection, tables were drawn up, considering the research variables. Only after acknowledgment and agreement by the Municipal Secretariat of Umari-Ce, the study protocol was submitted to the Ethics Committee for Research with Human Beings of the Faculdade Santa, according to CNS Resolution No. 466 of 201 with Opinion No. 5,110,876 of approval. RESULTS: The analysis of the variables showed deficiencies in the care provided, especially in relation to early recruitment and exams. Considering the sample, $65.5 \%$ were registered until the $12^{\text {th }}$ week of pregnancy, $47 \%$ underwent six or more consultations, 34\% reported age over 34 years, $62 \%$ were classified within the parameters for high gestational risk. The results showed an absence in the item "exams requested in the third trimester" for the studied group. CONCLUSION: The study reveals weakness in the early recruitment of pregnant women to start prenatal care, portraying the presence of high risk among the sample, as well as a discrepancy between the components requesting and carrying out rapid tests and laboratory tests.

KEYWORDS: Pregnancy; Prenatal care; Nursing care; Obstetric Nursing; Primary Health Care. 


\section{INTRODUÇÃO}

O desenvolvimento gestacional embora seja um fenômeno fisiológico natural é composto de alterações corporais, psicológicas e sociais singulares, que desencadeiam mudanças no dia-a-dia da mulher. É preciso refletir sobre os parâmetros e vínculos que rodeiam essas mulheres, pois influem fortemente na acessão ao pré-natal, no domínio da atenção e dos cuidados a serem desempenhados (ROCHA et al, 2019).

A morbimortalidade materna é um enorme problema de saúde publica, especialmente nos países subdesenvolvidos, contudo, é um agravo considerado intolerável nos dias de hoje, por ser correntemente prevenível. Apesar da diminuição da mortalidade materna ser um propósito mundial e está sucedendo com queda significativa, essa ainda se mantém em níveis elevados globalmente (ASSIS et al, 2020).

A atenção primária a saúde (APS) é retratada como um composto de intervenções de saúde no âmbito individual e coletivo, categorizando-se como a porta de entrada do Sistema Único de Saúde. Possui o designo de ofertar à comunidade acolhida por aquele serviço, uma atenção integral, agregada a promoção, prevenção e proteção à saúde, investigação, terapêutica e reparação de danos e agravos (MISQUITA et al, 2020).

O pré-natal é um recurso preventivo que compõe a APS, que busca através do acompanhamento contínuo, assegurar um sadio decorrer do ciclo gestacional e proporcionar o nascimento de um bebê saudável. Pesquisas revelam que um prénatal eficiente está interligado à restrição de términos perinatais desfavoráveis, como baixo-peso e imaturidade, além de reduzir a proporção de complicações obstétricas, como distúrbios hipertensivos, DM gestacional e mortalidade materna (MARQUES et al, 2020).

Masques et al. (2020) ainda afirma que, apesar dos elevados registros de consultas de pré-natal no Sistema Único de Saúde (SUS), é reduzido o percentual 
das mulheres que recebem acompanhamento pré-natal satisfatório. Reforçando que somente o crescimento da cobertura de consultas de supervisão no pré-natal não é suficiente para execução positiva da assistência prestada, ressaltando assim a importância da qualificação das consultas de pré-natal.

Para uma atenção pré-natal de qualidade, é necessário à união de estímulos na composição e administração dos serviços de saúde, uma educação continuada e um aprimoramento na aplicabilidade das tecnologias de saúde, para assim alcançar um acompanhamento favorável no período gestacional (SOUZA et al, 2020).

$O$ profissional de enfermagem tem alta relevância na efetivação desse serviço, o mesmo precisa estar apto e treinado para ofertar e gerenciar ações de promoção e prevenção à saúde, e prestar cuidado humanizado, integral e holístico as gestantes. Com isso, o enfermeiro pode traçar as metas e planejar as condutas de orientação para os atendimentos de pré-natal, mediante as fragilidades detectadas, determinando as mediações, instruções e direcionamento para serviços de referência, e ainda possibilitar a multidisciplinaridade das intervenções (SEHNEM et al, 2020).

É notório que há irregularidades em relação à organização, elaboração e programação das unidades básicas de saúde, enfatizam-se falhas na edificação, nos recursos humanos e materiais, e ainda dificuldade para a realização de exames e atraso no recebimento dos resultados. Erros esses, considerados entraves para os enfermeiros efetivar um cuidado assertivo, dado que, eles impossibilitam a assistência, a supervisão de maneira completa e o desfecho positivo dos problemas (NASCIMENTO et al 2020).

No entanto, destaca-se que um dos pontos fundamentais para a melhor oferta de serviços está pautada no perfil da população assistida, em que desvendar as características da população pode ser o elemento norteador para o planejamento direcionado e fiel das ações a serem desenvolvidas.

Nessa perspectiva o estudo buscou apresentar as características das gestantes atendidas em consultas de enfermagem ambulatoriais no período de 2011-2021. 


\section{METODOLOGIA}

Pesquisa documental, retrospectiva, por meio de consulta ao banco de dados do Sistema de Informação do Pré-natal (Sisprenatal), através do portal DATASUS (Departamento de Informática do Sistema Único de Saúde), no período de novembro de 2021, no setor de Informática da Secretaria Municipal de Saúde de Umari-CE.

A amostra foi não-probabilística por conveniência e consistiu de todas as gestantes cadastradas no Sisprenatal no período de 01 de janeiro de 2011 a 01 de janeiro de 2021 - não havendo assim critérios de exclusão.

Os dados foram obtidos através dos relatórios gerais gerados e emitidos pelo Sistema, referente década apresentada (2011/2021). Utilizamos como variáveis dependentes: número de gestantes cadastradas, quantidade de gestante cadastrada até a $12^{\text {a }}$ semana, faixa etária, etnia, presença ou ausência de risco gestacional, realização testes rápidos (sífilis e anti- HIV), e realização de exames laboratoriais (Hemograma, EAS, VRDL, sorologia para toxoplasmose, Glicemia em Jejum).

As informações obtidas a partir dos dados coletados tiveram caráter sigiloso, sendo as gestantes identificadas apenas por um código numérico do SISPRENATAL, o que torna impossível relacionar o nome do sujeito de pesquisa ao seu número. Ressalta-se que, de acordo com desenho de estudo, não houve entrevista com as participantes. O estudo foi submetido ao Comitê de Ética em Pesquisa com Seres Humanos da Faculdade Santa, conforme Resolução CNS № 466 de 2012, com parecer de aprovação nำ5.110.876.

Considerando a análise dos dados, as informações foram descritas em números absolutos e percentuais, apresentada em tabelas.

Os autores declaram inexistência de conflitos de interesse. 


\section{RESULTADOS E DISCUSSÃO}

As variáveis foram agrupadas em três blocos, a saber: o primeiro alude-se ao número de gestantes cadastradas, gestantes cadastradas até a $12^{\text {a }}$ semana de idade gestacional, e número de consultas realizadas; o segundo indica as características das gestantes (faixa etária, etnia e risco gestacional); e o terceiro compõe os exames e testes rápidos solicitados/realizados.

Tabela 1. Número de gestantes cadastradas, IG de cadastramento das Gestantes; número de consultas realizadas.

\begin{tabular}{llccc}
\hline \multicolumn{1}{c}{ Variáveis } & & Número & Porcentagem \\
\hline Quantidade de Gestantes cadastradas & & 338 & 100,0 \\
Quantidade de Gestantes cadastradas até a $12^{\underline{a}}$ & 222 & 65,6 \\
semana & & & 159 & 47,0 \\
Gestantes com 6 ou mais consultas & & 1723 & - \\
\hline Total de consultas realizadas & &
\end{tabular}

Fonte: SISPRENATALWEB/GESTANTES.

Tabela 2. Características das gestantes cadastradas.

\begin{tabular}{lccc}
\hline & Variáveis & Número & Porcentagem \\
\hline Idade & & \\
De 15 a 19 anos & 08 & 2,3 \\
De 20 a 24 anos & 61 & 18,0 \\
De 25 a 29 anos & 67 & 19,8 \\
De 30 a 34 anos & 85 & 25,1 \\
Acima de 34 anos & 117 & 34,6 \\
Total & $\mathbf{3 3 8}$ & $\mathbf{1 0 0 , 0}$ \\
\hline
\end{tabular}

\section{Raça/Cor}

Amarela

Branca

Preta

Parda

Sem informação

$6 \quad 1,7$

Total 


\begin{tabular}{lrr}
\hline Classificação de Risco da Gestante & & \\
Risco Habitual & 183 & 54,1 \\
Alto Risco & 142 & 42,0 \\
Sem informação & 13 & 3,8 \\
Total & $\mathbf{3 3 8}$ & $\mathbf{1 0 0 , 0}$ \\
\hline
\end{tabular}

Fonte: SISPRENATALWEB/GESTANTES.

Tabela 3. Exames laboratoriais e testes rápidos solicitados/realizados.

\begin{tabular}{|c|c|c|}
\hline Variáveis & Número & Porcentagem \\
\hline \multicolumn{3}{|l|}{$\begin{array}{l}\text { Quantidade de exames Solicitados } \\
\text { 10 Trimestre }\end{array}$} \\
\hline 2ㅇ Trimestre & 1918 & 69,6 \\
\hline $3^{\circ}$ Trimestre & 835 & 30,3 \\
\hline Total & 0 & 0,0 \\
\hline & 2753 & 100,0 \\
\hline \multicolumn{3}{|l|}{ Quantidade de exames Realizados } \\
\hline 10 Trimestre & 1305 & 63,8 \\
\hline 2ㅇ Trimestre & 453 & 22,1 \\
\hline 3o Trimestre & 287 & 14,0 \\
\hline Total & 2045 & 100,0 \\
\hline \multicolumn{3}{|l|}{ Testes rápidos para Sífilis } \\
\hline Solicitados & 39 & 55,7 \\
\hline Realizados & 31 & 44,2 \\
\hline Total & 70 & 100,0 \\
\hline \multicolumn{3}{|l|}{ Testes rápidos para HIV } \\
\hline Solicitados & 54 & 55,1 \\
\hline Realizados & 44 & 44,9 \\
\hline Total & 98 & 100,0 \\
\hline
\end{tabular}

Fonte: SISPRENATALWEB/GESTANTES.

Após análise da tabela 1, calculando a média entre as consultas realizadas e o número de gestantes cadastradas, obteve-se o total de 5 (cinco) consultas em média por gestante. Ficando assim, apenas $47,0 \%$ das mulheres, dentro do que é preconizado pelo ministério da saúde, que é de no mínimo 6 (seis) consultas de acompanhamento pré-natal.

As consultas de pré-natal são instrumentos de grande influência para a efetiva assistência do período gestacional, apresentando-se como relevante momento para orientar, planejar ações que se desdobrarão no decurso do período, detectar causas de risco, observar os parâmetros de saúde da mulher e do feto, tratar e prevenir 
disfunções em tempo propício. Desse modo, o profissional de enfermagem que atua na assistência ambulatorial das unidades básicas de saúde (UBS), mediante seu saber teórico-prático, deve estar vinculado de forma oportuna com a gestante e seus familiares, registrando suas intervenções de modo sistematizado. O enlace de todos os pontos contribuirá para desfechos maternos e perinatais mais acertados (TAVARES et al, 2019).

Analisado tal como, o cadastramento precoce, ou seja, até a $12^{\underline{a}}$ semana de gestação, sendo que $65,68 \%$ das gestantes tiveram início do acompanhamento gestacional dentro desse parâmetro recomendado, ficando assim 34,32\% das gestantes com início do acompanhamento gestacional ao fim do $1^{\circ}$ trimestre.

O Programa de Humanização no Pré-Natal e Nascimento (PHPN) estabelece que o pré-natal deva ser iniciado no primeiro trimestre, o mais precocemente possível após comprovação da gestação. Neste contexto, autores alegam que problemas na assistência ao pré-natal possam surgir com o início serôdio: redução do número de consultas, predisposição à falha na supervisão de exames, na imunização e escassez de orientações sobre todo o período gravídico puerperal (NASCIMENTO et al, 2016).

Reforçando estes achados, o estudo de NASCIMENTO et al (2016) ainda retrata que, o que conduz as mulheres a procurarem o acompanhamento do prénatal, é a relevância do programa para obter uma gestação saudável e o saber sobre o desenvolvimento sadio do feto. Porém, por muitas vezes, não existe estimulo próprio na mulher para iniciar o pré-natal, sendo ainda mais desafiador para o sistema de saúde, quando essa mulher esta inserida em um meio cultural onde o autocuidado e o cuidado do ambiente em que vive são considerados irrelevantes, exigindo empenho maior dos profissionais para a efetividade do pré-natal.

A atuação do enfermeiro é imprescindível no alcance dessa efetivação. O vínculo entre o profissional e as gestantes precisa ser estruturado ao longo dos atendimentos de enfermagem, com acolhimento humanizado, escuta qualificada e explanação de dúvidas, proporcionando laços de confiança. Esse elo, juntamente com as ações dos Agentes Comunitários de Saúde (ACS), com os serviços de ação social, o auxilio das pastorais e o envolvimento familiar, torna o cuidado mais ativo e 
incessante, repercutindo positivamente na confiabilidade do serviço para a gestante, facilitando sua adesão ao plano de cuidado (GONÇALVES et al, 2016).

Ao analisarmos a tabela número 2 , pode-se notar um maior índice de gestantes acima de 34 anos, apresentando $34,62 \%$ das gestantes cadastradas no SISPRENATAL do referido município.

Nas últimas décadas notou-se uma maior propensão nas mulheres em protelar a gestação. Diversos fatores estão envolvidos nesse processo como, estabilidade financeira, busca por aprimoramento profissional, diversidade de métodos contraceptivos e facilidade de alcance dos mesmos, aumentando consideravelmente os índices de gestações tardias em diversas partes do mundo, e correlacionado a isso o aumento também da incidência de partos cesarianos (TAKAGl et al, 2018).

Estudos apontam alguns fatores positivos relacionados à gravidez com idade superior a 35 anos, são eles, maior adesão ao aleitamento materno, constância emocional e financeira, ampliação de conhecimentos. Entretanto, os fatores de risco associados às gestações tardias se sobressaem. Riscos relacionados à mulher: aumento do ganho de peso, distúrbios metabólicos, doenças hipertensivas, fibromioma uterino e ruptura prematura de placenta; Complicações relacionadas ao concepto: anormalidades cromossômicas, prematuridade, presença de mecônio intraparto, desnutrição fetal, restrição de crescimento intrauterino, macrossomia fetal, sofrimento fetal, internação em UTI e óbito (SANTANA, 2018).

A idade materna não deve ser considerada como causa isolada para desencadear complicações gestacionais. Tem que ser encarada como indicador de risco, ou seja, é uma variante que abrange o mecanismo do processo fisiológico, no caso em questão, a gestação. Os estudiosos ainda não conseguem afirmar ao certo a idade em que os riscos são desencadeados. As variantes de idade modificam-se ao passar dos anos e conforme a localização dos estudos. Constatar os aspectos mais relevantes que podem ocasionar riscos maternos e fetais em gestantes com idade avançada contribui para uma assistência integral durante todo o período gravídico puerperal (ALMEIDA et al, 2019). 
Ainda na tabela 2 um valor que nos faz atentar é o percentual de $42,1 \%$ das gestantes em questão encontrar-se dentro da classificação de alto risco, valor bastante relevante em relação ao total cadastrado.

Ressaltamos que durante toda gestação, podem ocorrer complicações que tornam uma gestação normal em alto risco. O Brasil, por suas grandes dimensões e principalmente por possuir uma população com diversas características socioeconômicas e culturais, evidenciam-se fatores de risco para as várias regiões, dentre os quais podemos destacar: Características individuais e condições econômicas desfavoráveis; história reprodutiva anterior; doença obstétrica na gravidez atual e/ou intercorrências clínicas.

As necessidades de saúde do grupo de alto risco geralmente requerem técnicas mais especializadas, ainda que alguns casos possam ser sanados no nível primário de assistência, por meio de procedimentos simples, outros demandarão cuidado e oferta de ações a partir de uma equipe multiprofissional, ampliando assim as possibilidades de um desfecho favorável para o binômio mãe/filho.

A gravidez é considerada de alto risco quando a mulher apresenta alguma patologia ou agravo que prejudica a evolução do ciclo gravídico, podendo vir a causar complicações e até mesmo a morte materna. Com isso, torna-se fundamental a ligeira detecção das causas que acarretam complicações em uma gestação, com o intuito de identificar as possíveis intervenções a serem empregadas para prevenção das mesmas e reduções da Morbimortalidade Materna (COSTA et al, 2016).

Conhecer o perfil epidemiológico das gestantes de alto risco, acompanhadas nos centros de referência, emergi conhecimentos pertinentes para resolução e precaução de possíveis interferências no período gravídico-puerperal. Mediante esse contexto, pesquisas relatam que a assistência ao pré-natal é inevitável para assegurar o transcorrer sadio da gestação, parto e puerpério. $O$ acompanhamento do pré-natal eficiente e eficaz possibilita a diminuição de desfechos maternos e perinatais desfavoráveis (RUAS et al, 2020).

Ao observarmos as variáveis da tabela 3 , há discrepância em relação ao número de gestantes assistidas com o número de testes rápidos solicitados e realizados, o que nos incita a fazer uma análise sobre o fato apresentado, já que o município conta com oferta gratuita de sorologia para os testes em questão, nos 
fazendo refletir em o que impediria a realização dos mesmos e em como efetivar positivamente esse quesito, sendo ele tão essencial para o cuidado materno-infantil e ferramenta chave para detecção precoce do HIV e Sífilis.

Os Testes Rápidos (TR) para sífilis e HIV devem ser realizados de predileção no primeiro e terceiro trimestres de gestação, segundo orientação do Ministério da saúde, são práticos e de fácil execução. Acredita-se que com a inserção de medidas e condutas provenientes dos Programas e Políticas de saúde alcance-se melhorias nos indicadores de sífilis e HIV nas gestações, acarretadas por antecipação do diagnóstico, inicio da terapêutica adequada e intervenções para prevenção da transmissão vertical, isto devido a viabilização dos TR nos serviços de saúde (LOPES et al, 2016).

Nos dias atuais, as unidades de saúde ainda enfrentam desafios na oferta e realização dos $T R$, por falta de recursos materiais, assim como profissionais com saber insuficiente para tratar os resultados positivos, originando em inexatidão nas condutas recomendadas. Salientando a necessidade de remodelação dos mecanismos adotados e apoio a capacitação permanente dos profissionais (ARAÚJO et al, 2020).

A tabela 3 nos mostra disparidade além do mais nos exames solicitados em relação aos exames realizados. Sendo que dos 2.753 exames solicitados nas consultas de acompanhamento do pré-natal apenas 2.045 foram realizados, segundo informações contidas nos relatórios do Sisprenatal, essa baixa porcentagem de registro dos resultados de exames solicitados pode estar relacionado a falhas na alimentação do sistema de acompanhamento, como além do mais na própria realização dos mesmos, devido à escassez de recursos materiais no município, déficit financeiro das gestantes, filas de espera, atraso na devolutiva dos resultados e perda das amostras coletadas, causando grande prejuízo na detecção de agravos, que poderão repercutir em complicações futuras para o binômio mãefilho.

A realização dos exames preconizados durante o acompanhamento do ciclo gravídico é fundamental para assegurar uma assistência acertada, deste modo, promovendo a diligência de complicações e recurso terapêutico em tempo hábil, 
sucedendo na diminuição dos índices de morbimortalidade materna e neonatal (FERREIRA et al, 2017).

Exames auxiliam no rastreio e na resolubilidade de possíveis agravos. As causas que levam a suas solicitações precisam ser explicitadas à gestante. É oportuno suceder orientações antes e após a realização dos mesmos, ressaltando as vantagens e desvantagens, assim como distinguir o conhecimento e perspectivas da gestante com relação aos resultados (CAVALCANTE et al, 2016).

Pesquisas apontam que o baixo nível educacional, gravidez na adolescência, baixas condições socioeconômicas, desestrutura familiar e consumo de drogas são amostras de impedimentos que resultam na baixa admissão ao pré-natal, abrangendo inclusive a realização integral dos exames (SALDANHA, 2020).

Para potencializar a assistência ao pré-natal no que se refere à solicitação de exames, uma boa estruturação física, aperfeiçoamento adequado das ferramentas tecnológicas e insumos suficientes são cruciais para execução das intervenções planejadas, favorecendo assim a efetividade da assistência prestada (DIAS et al, 2017)

\section{CONCLUSÃO}

O estudo revela fragilidade na captação precoce das gestantes para início do acompanhamento pré-natal, retratando a presença de alto risco entre a amostra, assim como discrepância entre os componentes solicitação e realização de testes rápidos e exames laboratoriais.

Para melhor efetivação das políticas e programas relacionados à saúde da mulher, especificamente durante o ciclo gravídico sugerimos monitoramento contínuo das ações, para fortalecer assim a saúde do binômio mãe/filho no município estudado. 


\section{REFERÊNCIAS BIBLIOGRÁFICAS}

ALMEIDA, Bruna Bergamini Pereira de; MORALES, Júlia Delli Colli. Idade Materna e Resultados Perinatais na Gestação de Alto Risco. 2019.

ARAÚJO, Túlio César Vieira de; SOUZA, Marize Barros de. Adesão das equipes aos testes rápidos no pré-natal e administração da penicilina benzatina na atenção primária. Revista da Escola de Enfermagem da USP, v. 54, 2020.

CAVALCANTE, Kariane Omena Ramos et al. Exames de rotina no pré-natal: solução ou problema?. Rev. enferm. UFPE on line, p. 1415-1422, 2016.

DALLA COSTA, Lediana et al. Perfil epidemiológico de gestantes de alto risco. Cogitare Enfermagem, v. 21, n. 2, 2016.

DIAS, Cristiano Leonardo de Oliveira; SILVA JUNIOR, Renê Ferreira da; BARROS, Sônia Maria de Oliveira. Análise da qualidade da assistência pré-natal no âmbito da Estratégia de Saúde da Família. Rev. enferm. UFPE on line, p. 2279-2287, 2017.

DOS SANTOS NASCIMENTO, Luana Carla et al. Perspectiva dos enfermeiros sobre a assistência pré-natal no âmbito da Estratégia Saúde da Família. Revista de Enfermagem da UFSM, v. 10, p. 44, 2020.

FEITOSA-ASSIS, Ana Isabela; SANTANA, Vilma Sousa. Ocupação e mortalidade materna. Revista de Saúde Pública, v. 54, p. 64, 2020.

FERREIRA, Helder et al. Rotina de exames na atenção ao pré-natal após a implantaçãodo programa rede mãe paranaense. Rev. enferm. UFSM, p. 1-15, 2017.

GONÇALVES, Mirela Dias; KOWALSKI, Ivonete Sanches Giacometti; SÁ, Ana Cristina. Atenção ao pré-natal de baixo risco: atitudes dos enfermeiros da estratégia saúde da família [Low-risk antenatal care: family health strategy nurses' attitudes]. Revista Enfermagem UERJ, v. 24, n. 6 , p. 18736, 2016.

LOPES, Ana Cristina Martins Uchoa et al. Implantação dos testes rápidos para sífilis e HIV na rotina do pré-natal em Fortaleza-Ceará. Revista Brasileira de Enfermagem, v. 69, n. 1, p. $62-$ 66, 2016.

MARQUES, Bruna Leticia et al. Orientações às gestantes no pré-natal: a importância do cuidado compartilhado na atenção primária em saúde. Escola Anna Nery, v. 25, n. 1, 2021.

MISQUITA, Mirelly Shatilla et al. Atendimento de gestantes na atenção primária a saúde pela enfermagem durante a pandemia do SARS-COV-2. Nursing (São Paulo), v. 23, n. 269, p. 47234730, 2020.

NASCIMENTO, Vagner Ferreira do et al. Percepção de puérperas sobre as primeiras consultas de pré-natal no interior de Mato Grosso. Rev. enferm. UFPI, p. 46-51, 2016.

ROCHA, Carolina Gabriele Gomes da et al. Determinantes sociais da saúde na consulta de enfermagem do pré-natal. Rev. enferm. UFPE on line, p. [1-8], 2019.

RUAS, Carla Alaíde Machado et al. Perfil e distribuição espacial da mortalidade materna. Revista Brasileira de Saúde Materno Infantil, v. 20, n. 2, p. 385-396, 2020. 
SALDANHA, Bruna Lopes. Dificuldades enfrentadas por gestantes adolescentes em aderir ao pré-natal. Revista Eletrônica Acervo Saúde, v. 12, n. 9, p. e4160-e4160, 2020.

SANTANA, Bruna Luiza Costa. Gestação acima de 35 anos, resultados perinatais e via de parto no Brasil: uma revisão integrativa. 2018.

SEHNEM, Graciela Dutra et al. Consulta de pré-natal na atenção primária à saúde: fragilidades e potencialidades da intervenção de enfermeiros brasileiros. Revista de Enfermagem Referência, n. 1, p. e19050, 2020.

SOUZA, Rodrigo Ayres de et al. Avaliação de qualidade da assistência pré-natal prestada pelo enfermeiro: pesquisa exploratória. Online braz. j. nurs. (Online), 2020.

TAKAGI, Maira Maiko et al. Resultados perinatais em gestantes acima de 35 anos. Arquivos Médicos dos Hospitais e da Faculdade de Ciências Médicas da Santa Casa de São Paulo, v. 55, n. 3, p. 108-114, 2018.

TAVARES, Daniel Soares et al. Construção e validação de um histórico de Enfermagem para consulta pré-natal. Enferm. foco (Brasília), p. 35-42, 2019. 